Mappemonde

Revue trimestrielle sur l'image géographique et les formes du territoire

$126 \mid 2019$

Varia

\title{
Des colporteurs syriens dans la mondialisation
}

Syrian street vendors and globalisation

Los vendedores ambulantes sirios y la globalización

David Lagarde

\section{OpenEdition}

Journals

Édition électronique

URL : http://journals.openedition.org/mappemonde/1363

DOI : 10.4000/mappemonde.1363

ISSN : 1769-7298

Éditeur

UMR ESPACE

Référence électronique

David Lagarde, «Des colporteurs syriens dans la mondialisation », Mappemonde [En ligne], 126 | 2019, mis en ligne le 01 avril 2019, consulté le 11 novembre 2019. URL : http://journals.openedition.org/ mappemonde/1363 ; DOI : 10.4000/mappemonde.1363

Ce document a été généré automatiquement le 11 novembre 2019.

\section{(c) (i) (2)(2)}

La revue Mappemonde est mise à disposition selon les termes de la Licence Creative Commons Attribution - Pas d'Utilisation Commerciale - Partage dans les Mêmes Conditions 4.0 International. 


\title{
Des colporteurs syriens dans la mondialisation
}

\author{
Syrian street vendors and globalisation \\ Los vendedores ambulantes sirios y la globalización
}

\author{
David Lagarde
}

\section{Introduction}

1 Lors d'un terrain de recherche doctorale débuté à Amman en 2014, j'ai fait la connaissance de réfugiés syriens originaires de Deir Mqaren, un village situé dans la vallée du Barada, entre Damas et la frontière libanaise (figure 1). Avant le début du conflit, la majorité d'entre eux avait l'habitude de circuler régulièrement entre cette localité, le Liban et la Jordanie, afin de vendre des mokasarat ${ }^{1}$, des fruits secs et différentes sortes de friandises qu'ils achetaient en Syrie, pour les revendre ensuite à l'étranger.

Inspiré par les travaux de plusieurs contributeurs de ce numéro spécial (Pliez, 2007 ; Pliez et Belguidoum, 2012 ; Bouhali, 2015 ; Doron, 2015 ; Choplin et Pliez, 2018), je me suis appliqué à retracer l'émergence du réseau commercial des colporteurs de Deir Mqaren, sa diffusion spatiale, ainsi que la provenance des denrées vendues par ces marchands en Jordanie. Si l'ambition de cette recherche doctorale visait principalement à souligner l'influence de ces circulations sur les trajectoires actuelles des réfugiés originaires de cette bourgade (Lagarde et Doraï, 2017), étudier ce réseau marchand et migratoire à partir d'une approche longitudinale m'a également permis de témoigner de l'ancienneté de ce type de pratiques au Proche-Orient, tout en mettant en exergue l'évolution des filières d'approvisionnement des colporteurs faisant l'objet de ma thèse (Lagarde, 2018). La contribution proposée ici entend donc revenir sur la façon dont cette activité, fondée à l'origine sur une production agricole exclusivement locale, est progressivement venue se greffer sur des réseaux transnationaux du commerce mondial, en réponse à des contraintes structurelles nationales et régionales. Ce faisant, il s'agira aussi de dévoiler en filigrane l'une des multiples facettes que sont susceptibles 
d'adopter les «mondialisations discrètes» (Choplin et Pliez, 2015, 2016), qui, par l'action d'une multiplicité d'acteurs peu voire pas visibles, contribuent à relier entre elles des villes et des bourgades des Suds, situées à l'écart des grands centres décisionnels et économiques les plus médiatisés de la planète.

Figure 1. Localisation du village de Deir Mqaren

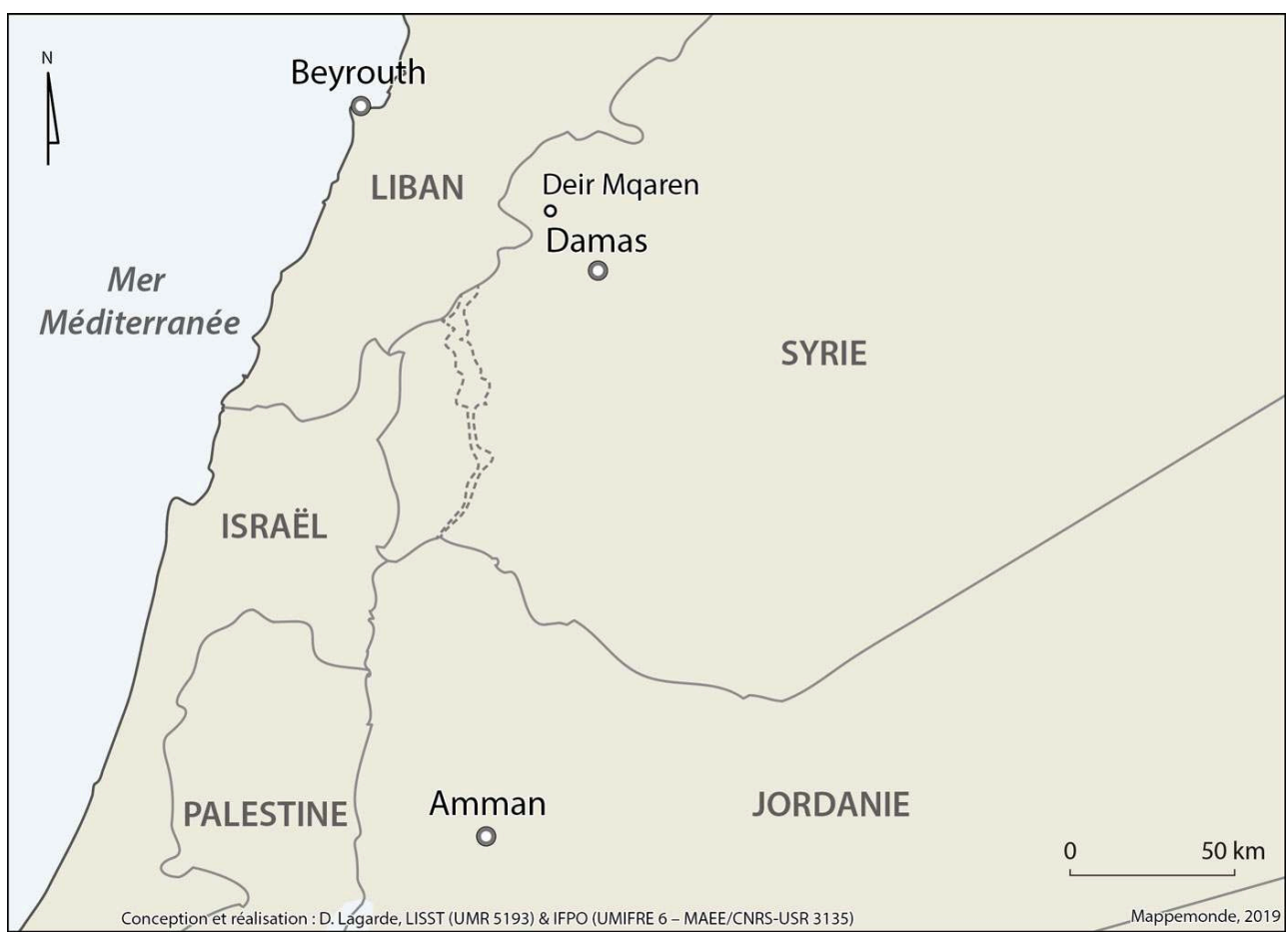

D. Lagarde

\section{Approche méthodologique}

En décidant d'analyser les circulations des habitants du village de Deir Mqaren, l'une des intentions de ma thèse était de proposer une géographie des migrations internationales empreinte d'une forte sensibilité ethnographique, seule à même de pouvoir illustrer de manière tangible le «continuum " (Richmond, 1993) existant entre les flux actuels de réfugiés et les mobilités économiques antérieures au conflit (Gehrig et Monsutti, 2003). Cela m'a amené à privilégier le « point de vue de l'autonomie ${ }^{2}$ » (Ma Mung, 2009), afin d'étudier la façon dont les acteurs et les groupes sociaux peuvent chercher à aménager par et pour eux-mêmes les conditions de leurs mobilités, que celles-ci soient motivées par des facteurs d'ordre économique et/ou sécuritaire. Privilégier le point de vue de l'autonomie revient finalement à placer les expériences individuelles au centre de l'analyse. Dans cette perspective, il m'est apparu primordial de focaliser mon attention sur les récits de vie des exilés de Deir Mqaren. Pour cela, j'ai cherché à reconstituer à partir d'une combinaison d'écoute attentive et de questionnements plus précis, la structure diachronique des évènements biographiques de 24 personnes originaires de ce village, désormais installées en Jordanie. Ce panel de 24 individus est composé de 7 femmes $^{3}$ et 17 hommes. Parmi ces derniers, l'intégralité a effectué le métier de colporteur au moins une fois dans sa vie, pour des périodes allant de quelques mois à plusieurs années. Notons par ailleurs que parmi ces 17 individus, 4 
n'avaient jamais exercé ce travail avant de venir s'installer en famille à Amman, à la suite de l'éclatement du conflit en Syrie.

Cette approche profondément qualitative m'a rapidement conduit à questionner les caractéristiques du « système de mobilité » (Cortes, 1998) des habitants de ce village sur le temps long des migrations syriennes au Proche-Orient. Pour ce faire, je me suis attaché à collecter des récits rassemblant des mémoires transgénérationnelles, en mobilisant des techniques d'enquêtes inspirées du courant de l'histoire orale (Descamps, 2006). Cet aspect de la recherche m'a permis d'identifier les principales caractéristiques de la vie quotidienne des habitants de Deir Mqaren depuis le début du $\mathrm{XX}^{\mathrm{e}}$ siècle, en particulier en termes de saisonnalités de leurs itinéraires marchands. Les entretiens semi-directifs que j'ai conduits auprès des 24 enquêtés ${ }^{4}$ m'ont aussi offert la possibilité de saisir les spécificités des circulations plus contemporaines reliant Deir Mqaren aux principales villes de Jordanie ; soit entre le milieu des années 1980 et la fin de mon enquête jordanienne, en décembre 2015. Ces techniques d'entretien ont également été combinées avec des phases d'observation participante, conduites en 2014 et 2015 au sein des logements des exilés de Deir Mqaren dans le royaume hachémite, et dans une moindre mesure sur leurs lieux de vente jordaniens, en particulier à Amman. De cette façon, j'ai progressivement réussi à identifier l'évolution de leurs sources d'approvisionnement en marchandises et les contours des réseaux commerciaux dans lesquels ils se sont inscrits depuis le début du siècle dernier.

\section{Évolution de la provenance des mokasarat vendues en Méditerranée}

5 Si les premières mentions du commerce de fruits à coque et de fruités séchés en Méditerranée remontent à l'Antiquité, c'est véritablement à l'époque médiévale que ce type de produit a commencé à faire l'objet d'un commerce florissant, alimentant les échanges entre les différentes places marchandes de la région (Puig, 2005). Depuis le Moyen-Âge, les amandes, les noix, les pignons de pin, les noisettes, les pistaches, les figues ou encore les abricots font partie des principales variétés produites et échangées sur les pourtours du bassin méditerranéen. Néanmoins, au cours du $\mathrm{XX}^{\mathrm{e}}$ siècle, l'accélération de la libéralisation des échanges commerciaux à l'échelle mondiale a entraîné une importante recomposition des filières d'approvisionnement, une modification des modes de consommation, ainsi qu'une évolution de la demande des consommateurs.

6 Aujourd'hui, ces produits sont consommés quotidiennement au sud et à l'est de la Méditerranée. Si les pays du pourtour méditerranéen continuent de produire d'importantes quantités de figues et d'abricots secs, et que la Turquie demeure de très loin le premier producteur de noisettes ${ }^{5}$ au monde, l'essentiel des mokasarat provient désormais d'Afrique, d'Amérique et d'Asie. Cette tendance s'est d'ailleurs traduite par une diversification des fruits à coque composant ces mélanges. En effet, cela fait déjà plusieurs décennies que des cacahuètes, des noix de cajou et de macadamia se sont invitées dans ces assortiments, tant prisés des habitants du pourtour méditerranéen. Les mokasarat proposés sur les étals des souks du Maghreb, du Proche-Orient et des bazars des Balkans proviennent donc désormais des quatre coins du globe. En Syrie, jusqu'au début des années 1980, les habitants de Deir Mqaren continuaient toutefois de vendre exclusivement des noix, des amandes, des noisettes, des raisins, des figues ou 
encore des abricots séchés provenant de leurs vergers, situés sur les rives du Barada et le plateau faisant face au village.

\section{Arboriculture et circulations marchandes au début du $\mathrm{XX}$ siècle}

7 Deir Mqaren est situé dans le massif de l'Anti-Liban, à une vingtaine de kilomètres au nord-ouest de Damas. Ce village est traversé par le Barada, une rivière endoréique prenant sa source dans la plaine de Zabadani (figure 2). Pendant des siècles, ce cours d'eau constituait une ressource essentielle pour les populations de la vallée éponyme, qui vivaient principalement de l'arboriculture fruitière (Thoumin, 1936). Au début du $\mathrm{XX}^{\mathrm{e}}$ siècle, les habitants de Deir Mqaren vivaient au rythme du calendrier agricole et des itinéraires saisonniers que suivaient les paysans colporteurs afin de vendre leur récolte à Damas, au Liban, en Palestine mandataire et en Transjordanie. Grâce à la liberté de déplacement dont bénéficiaient alors les habitants de la région à l'échelle du Bilad esh-Sham ${ }^{6}$, les hommes de Deir Mqaren pouvaient se rendre librement dans ces contrées, pour y écouler une partie de leur production, mise à sécher à la fin de l'été et au début de l'automne. L'un des enquêtés, Bachar ${ }^{7}$, revient sur les spécificités de ces circulations saisonnières. "Mon père (...) était le propriétaire de nombreux terrains agricoles à Deir Mqaren, mais cela ne l'empêchait pas pour autant de travailler en étroite collaboration avec les autres habitants du village. (...) L'été, ils étaient occupés par la récolte des fruits dans les vergers, qui commence dès le mois de mai ». À la fin de l'automne, les hommes du village chargeaient leurs ânes des stocks de fruits à coque et de fruits séchés au soleil durant les mois précédents, avant de se rendre en groupe au Liban, pour y vendre une partie de leur production. À en croire Bachar, leurs principaux lieux de vente se situaient dans les environs de Bhamdoun, Jbeil et Tyr. « Ils privilégiaient en particulier les villages et les quartiers des villes où résidaient les chrétiens qui leur achetaient des fruits secs et des fruits à coque en prévision des fêtes de Noël. Puis une fois leur chargement écoulé, ils reprenaient la direction du village pour refaire le plein de marchandise, avant de repartir vers la fin du mois de janvier en direction de la Palestine et de (l'actuelle) Jordanie. Ce circuit les amenait jusqu'à Jérusalem, où ils laissaient leurs ânes dans des étables et chargeaient leurs kharij ${ }^{8}$ pour vendre leur marchandise à pied, dans les villages des alentours, comme continuent de le faire aujourd'hui leurs descendants. Ils remontaient ensuite vers Deir Mqaren en écoulant le reste de leur production dans les villes et les villages qu'ils traversaient " (figure 3). 
Figure 2. La plaine de Zabadani et la vallée du Barada d'après une carte de Richard Thoumin (1936)

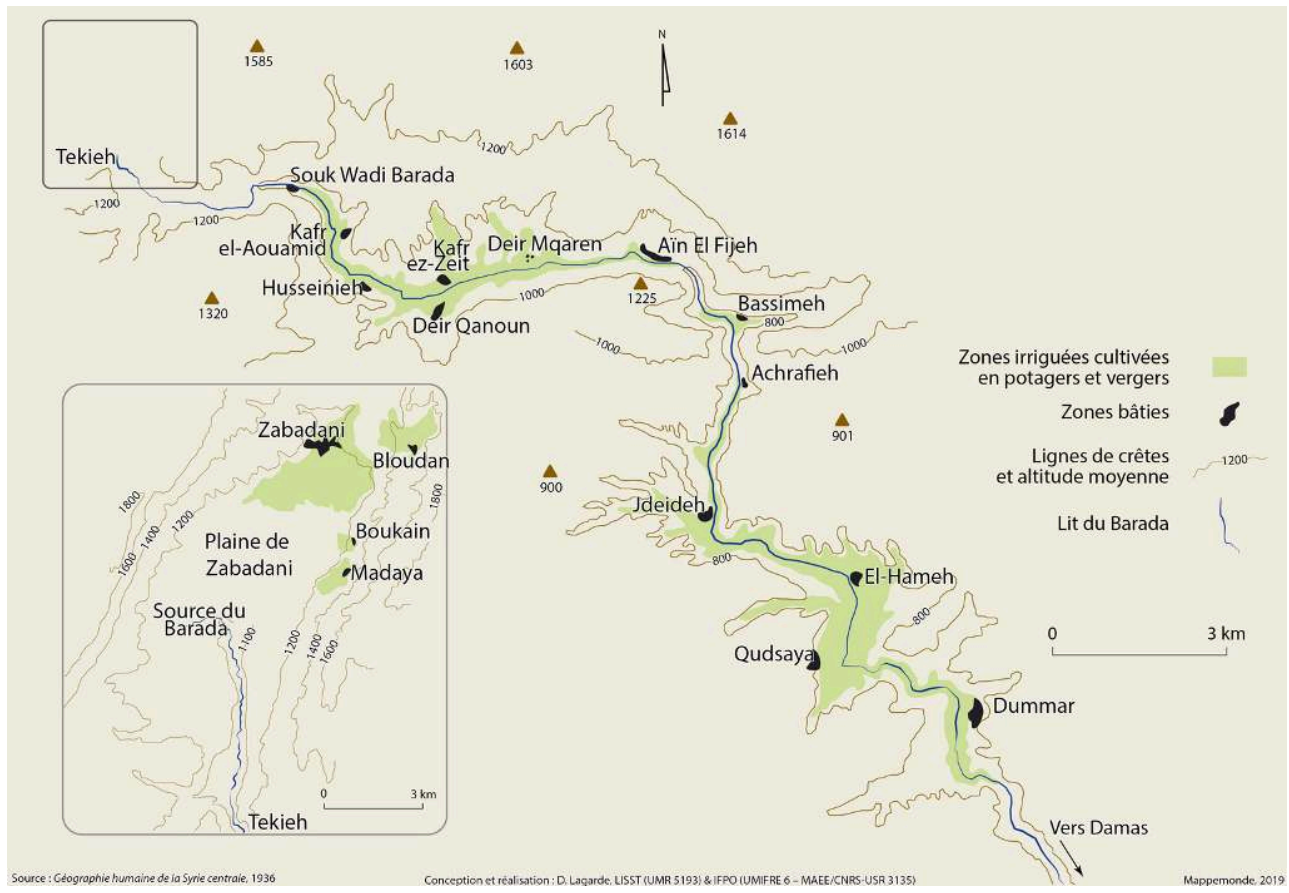

Sources : D. Lagarde, d'après Richard Thoumin, Géographie humaine de la Syrie centrale (1936) 
Figure 3. Itinéraires saisonniers des marchands de Deir Mqaren durant la première moitié du XXe siècle

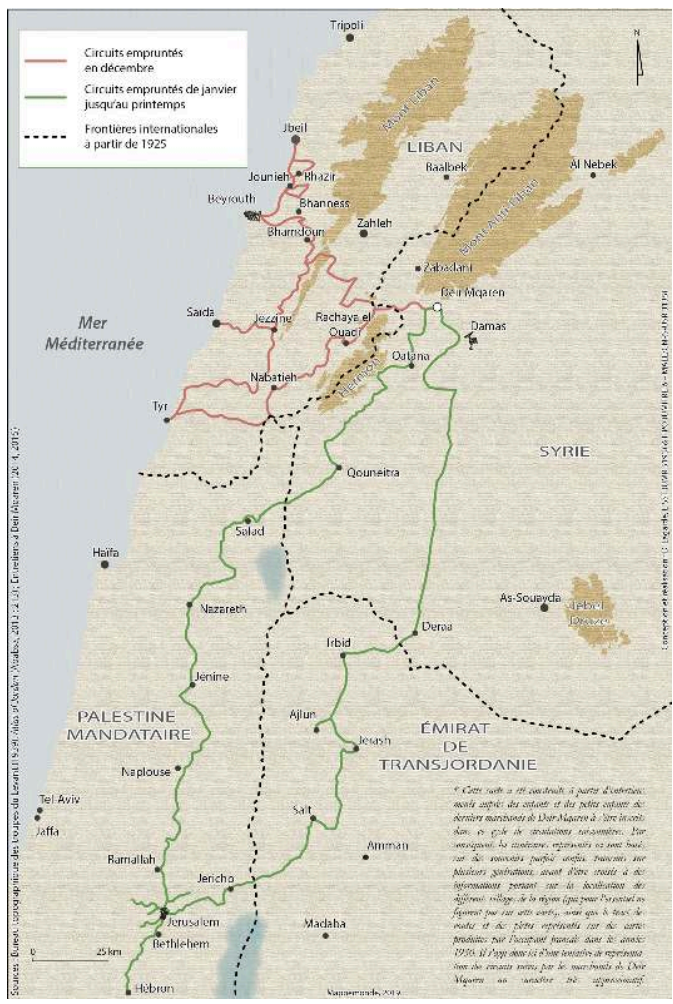

Cette carte a été construite à partir d'entretiens menés auprès des enfants et petits-enfants des derniers marchands de Deir Mqaren à s'être inscrits dans ce cycle de circulations saisonnières. Par conséquent, les itinéraires représentés ici sont basés sur des souvenirs parfois confus, transmis sur plusieurs générations, avant d'être croisés à des informations portant sur la localisation des différents villages de la région (qui pour l'essentiel ne figurent pas sur cette carte), ainsi que le tracé des routes et des pistes représentées sur des cartes produites par l'occupant français dans les années 1930. II s'agit ici d'une tentative de représentation des circuits suivis par les marchands de Deir Mqaren au caractère très approximatif.

Sources : Bureau topographique des troupes du Levant 1939 ; Atlas de Jordanie (Ababsa, 2013, p. 213) ; entretiens avec les vendeurs de Deir Mqaren $(2014,2015)$

Ces pratiques et ces mobilités, dont l'origine précise demeure impossible à dater, semblent avoir posé les jalons d'un système marchand ayant constitué une source centrale de revenus pour plusieurs générations de villageois. Les instigateurs de ces circulations marchandes ont ainsi ouvert des routes commerciales, reliant la localité de Deir Mqaren à d'autres points de vente et de sociabilité ailleurs dans la région. En accumulant des savoir-faire leur permettant d'écouler une production agricole locale, ils ont également développé des compétences circulatoires qui se sont transmises sur plusieurs générations (Tarrius, 1996), favorisant par là même les modalités «d'autonomisation» (Missaoui et Tarrius, 2006) de ce groupe face aux régimes centralisateurs et autoritaires qui se sont succédés à la tête de la Syrie à partir des années 1960. Leurs descendants ont toutefois dû faire preuve d'une grande capacité d'adaptation afin de faire perdurer ce réseau marchand, dans un contexte politique et économique souvent défavorable aux habitants de la vallée du Barada. En effet, si à l'écoute des descriptions empreintes de nostalgie - et comprenant sans nul doute une large part d'idéalisation - que la population de Deir Mqaren se transmet de génération en génération, le paysage et le rythme de vie de cette localité syrienne semblent être restés les mêmes jusqu'au tournant $\mathrm{du} \mathrm{XX}^{\mathrm{e}}$ siècle, plusieurs contraintes d'ordre 
structurel sont ensuite venues remodeler en profondeur les caractéristiques de cette activité agricole et commerciale.

\section{Des orientations politiques fatales au secteur agricole de la vallée}

En 1948, les violences qui suivent la création de l'État d'Israël marquent le premier acte du conflit israélo-arabe et amènent les autorités de l'État hébreu à appliquer un contrôle très strict des passages frontaliers (Derriennic, 1974). Pour les paysans colporteurs de Deir Mqaren, ces mesures se traduisent par une perte significative de leurs zones de commercialisation, engendrant une première reconfiguration spatiale de leur réseau marchand, avec un recentrement de leurs activités sur le Liban et le territoire du nord de la Jordanie. En 1963, le parti Baas s'empare ensuite du pouvoir et engage la Syrie dans une série de grandes réformes agraires, allant de pair avec un rapide développement industriel et un renforcement constant du pouvoir de l'armée, en particulier à partir de l'arrivée au pouvoir du général Hafez al-Assad ${ }^{9}$ (Longuenesse, 1979; Seurat, 1979; Picard, 2013). Ces grandes orientations politico-militaires engendrent un phénomène de centralisation important, à l'origine de plusieurs vagues de migrations internes. Ainsi, durant toute la seconde moitié du $\mathrm{XX}^{\mathrm{e}}$ siècle, l'agglomération de Damas fait l'objet d'une pression démographique constante. Entre les recensements de 1960 et 2004, la capitale passe en effet d'environ 530000 à 3,5 millions d'habitants. Sur le plan urbanistique, ce phénomène se traduit par un grignotage rapide des terres agricoles de la Ghouta ${ }^{10}$, sur lesquelles se multiplient les camps militaires, les zones industrielles, ainsi que d'innombrables logements au statut informel (Balanche, 2009). Les conséquences écologiques et sanitaires de cette explosion urbaine et démographique se révèlent désastreuses (Bianquis, 1977; Balanche, 2006) bien au-delà des seuls environs immédiats de la ville de Damas (Châtel, 2014). En effet, dans les années 1990, les besoins en eau de la capitale deviennent tels que « les autorités prirent la décision de percer une série de 120 puits de forage autour de la source du Barada " (Châtel et Raba'a, 2014) pour l'acheminer jusqu'à Damas par une conduite forcée traversant la vallée. Si cette mesure permet de répondre provisoirement au risque de pénurie d'eau pour les Damascènes, elle marque également le quasi-tarissement de la rivière en période d'étiage. Enfin, au nom de son combat contre «l'ennemi sioniste » et en s'appuyant "sur les bases de deux lois adoptées en 1958 et 1983 permettant au gouvernement de réquisitionner des terres pour des projets d'intérêt public» (Châtel et Raba'a, p. 12), plusieurs centaines d'hectares sont confisquées par le Ministère de la Défense dans la vallée du Barada. Pour les paysans de Deir Mqaren, cette mesure se solde par la perte de leurs vergers situés au sommet des gorges faisant face au village (figure 4). 


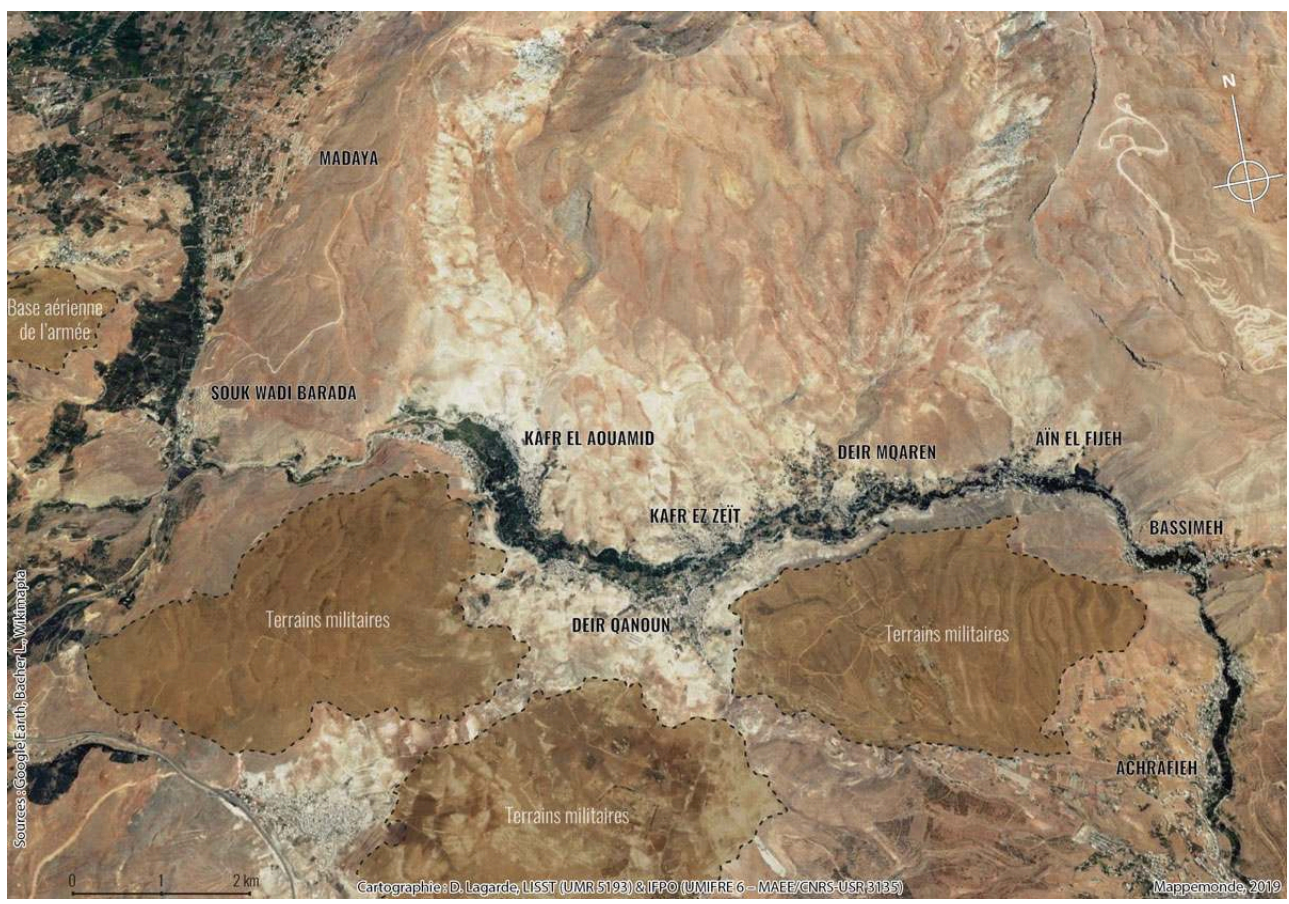

Un village situé dans une vallée stratégique et fortement militarisée.

Sources: Image satellite : Google Earth. Localisation des terrains militaires : entretien avec Bachar L. et Wikimapia. Cartographie : David Lagarde

10 Entre le début des années 1970 et la fin de la décennie 1980, les confiscations de terres agricoles, conjuguées à l'assèchement progressif du Barada, portent un coup d'arrêt fatal au secteur agricole de la vallée. Les rendements deviennent si faibles à Deir Mqaren que les revenus tirés de l'agriculture ne suffisent plus à faire vivre la population du village, en constante augmentation depuis le début du siècle ${ }^{11}$. Face à cette situation, les villageois se voient désormais contraints d'accéder à des sources de revenus alternatives.

\section{Vers une connexion progressive à des filières d'approvisionnement internationales}

Vers le milieu des années 1980, le cruel manque d'opportunités d'emplois dans la vallée incite plusieurs hommes de Deir Mqaren à se rapprocher de grossistes damascènes implantés dans la Ghouta. Leur objectif: se procurer ailleurs des produits qu'ils cultivaient encore eux-mêmes localement quelques années auparavant. Ces individus délaissent donc définitivement les itinéraires saisonniers empruntés depuis des décennies par les paysans colporteurs du village les ayant précédés, afin de s'inscrire à la place dans un système de migrations circulaires qui, jusqu'à l'éclatement du conflit syrien, amenait plusieurs centaines de personnes de Deir Mqaren à se rendre régulièrement dans les principales villes du Liban et de Jordanie, afin d'y revendre plus cher des fruits à coque et des friandises achetés à bas prix en Syrie. D’après Karam, un grossiste jordanien qui commerce avec les colporteurs du village depuis 1992, les mokasarat et les fruits secs que ces derniers ramenaient à l'époque de Syrie provenaient essentiellement de la région d'Alep, de Turquie, mais aussi d'autres régions du monde ${ }^{12}$. 
Ils se procuraient également des sucreries produites dans des usines de Damas, tandis que certains vendeurs en achetaient à des prix plus compétitifs directement en Jordanie, par l'intermédiaire de Karam. Enfin, il arrivait aussi à certains marchands d'acquérir une partie de ces denrées dans des magasins détaxés situés au postefrontière qui sépare la Syrie du royaume hachémite.

12 Au début de ce cycle de migrations circulaires, initié à partir du milieu des années 1980, les personnes impliquées dans ce commerce se contentent de vendre leur marchandise dans la ville d'Amman, et séjournent systématiquement à l'hôtel al-Hamra, un établissement bon marché situé au cœur du souk de la ville basse (figure 5). Durant toute la première moitié des années 1990, les capitaux injectés dans l'économie du royaume par les quelque 300000 Jordaniens de retour du Golfe après l'invasion du Koweït par l'Irak stimulent le secteur de l'immobilier. De nouveaux quartiers se développent à l'ouest de la capitale, tandis que les nouvelles échoppes commerçantes se multiplient dans les quartiers populaires du centre-ville. L'activité de vente ambulante devient alors de plus en plus florissante, incitant un nombre croissant d'habitants de Deir Mqaren à se lancer dans ce commerce, certains abandonnant même leur emploi en Syrie pour embrasser une carrière de colporteur. Les derniers arrivés investissent de nouveaux secteurs de vente dans la capitale, mais également dans la ville d'Irbid, qui connaît elle aussi une croissance soutenue à l'époque. Progressivement, les marchands se rendent aussi dans d'autres villes du pays, à Zarqa, Madaba, Jerash, Tafileh et Maan, tout en quadrillant les localités environnantes, à pied ou en voiture (figure 6). Durant plusieurs décennies, ces colporteurs ont ainsi fait vivre leurs familles restées au village en multipliant les allers-retours entre Deir Mqaren et leurs différents secteurs de vente en Jordanie, jusqu'à ce que le conflit syrien vienne affecter la fluidité de ces circulations marchandes transfrontalières. 
Figure 5. Quartiers du centre de l'agglomération d'Amman. Quartiers des souks de la ville basse

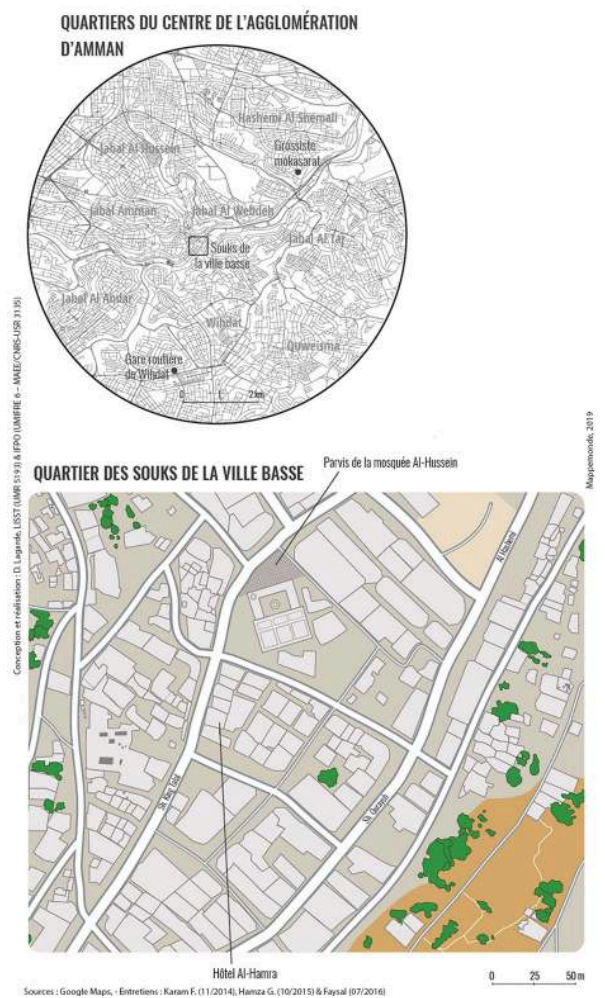

Conception et réalisation : David Lagarde. Sources : Google Maps ; entretiens avec Karam F. sur les routes de Jordanie (novembre 2014), Hamza G. à Irbid (octobre 2015) et Faysal à Dortmund (Juillet 2016)

Figure 6. Un réseau marchand transfrontalier à l'origine d'un système de mobilité



Conception et réalisation : David Lagarde. Sources : enquêtes de terrain en Jordanie (automnes 2014 et 2015) 


\section{Un réseau marchand synonyme de ressource en temps de crise}

13 À partir de mars 2011, dans la foulée des premières manifestations observées dans la ville de Deraa, un mouvement de contestation pacifique contre le régime de Bachar alAssad gagne d'autres villes et bourgades de Syrie. Malgré quelques interrogatoires parfois prolongés aux check-points dressés sur les routes du sud du pays ou au niveau du poste-frontière de Nassib/Jaber, les colporteurs de Deir Mqaren continuent autant que possible leurs allers-retours avec la Jordanie. Mais au mois de février 2012, alors que l'insurrection s'amplifie dans la vallée du Barada, le régime commence à s'en prendre de manière répétée aux habitants du village, qui sont pris pour cible par des tirs de mortier tirés depuis les terrains militaires situés sur les plateaux environnants (figure 4). Dans un tel contexte, les colporteurs deviennent de plus en plus réticents à l'idée de quitter leurs familles et cessent pour la plupart de se rendre à l'étranger pour travailler.

Le mois de juillet correspond ensuite à une multiplication des violences dans le reste du pays. C'est notamment le cas dans les centres-villes d'Alep et de Damas, les deux poumons politiques et économiques de la Syrie, restés jusqu'alors relativement épargnés par les violences. Si cette situation fait grossir les rangs des Syriennes et des Syriens qui, depuis plusieurs mois déjà, commencent à trouver refuge dans les pays limitrophes (Lagarde, 2019), elle entraîne également le pays dans une profonde crise économique. Alors que la Syrie s'enlise dans un conflit violent et que les frontières deviennent de plus en plus difficiles à franchir, la vente ambulante à l'étranger apparaît de plus en plus comme l'unique source de revenus envisageable pour une part croissante de la population de Deir Mqaren. En effet, les colporteurs qui ont cessé leur activité quelques mois auparavant voient leurs économies s'épuiser rapidement. Certains d'entre eux prennent donc la décision de partir s'installer seuls ou en famille en Jordanie. À la même période, la dégradation rapide de l'économie syrienne et la multiplication des licenciements qui en découlent amènent aussi des habitants du village employés dans d'autres secteurs d'activités en Syrie à se reconvertir dans le colportage de menues denrées alimentaires, à l'instar de Mahmoud. "Même si la vente ambulante rapportait pas mal d'argent à certains de mes proches, j'ai toujours préféré conserver mon emploi en Syrie pour continuer d'y vivre de manière permanente, car l'idée de passer des semaines entières loin de ma famille et de mes amis ne m'emballait pas vraiment. Mais en 2012, avec les problèmes dans le pays, le business a commencé à ralentir et mon patron m'a finalement licencié au moment où les combats ont touché la capitale. Quand je me suis retrouvé au chômage, (...) la vente ambulante m'est en fait apparue comme une évidence; tout du moins comme l'option la plus simple pour accéder rapidement à un revenu ».

15 Les hommes qui découvrent la vente ambulante à partir de 2012 rejoignent leurs cousins, leurs frères et leurs amis du village dont ils se sentent affectivement le plus proches. En s'agrégeant à un collectif auquel ils sont liés par des appartenances villageoises et/ou familiales, caractérisé par un fort esprit de solidarité et disposant de ressources internes au groupe, leur installation dans le tissu urbain des villes dans lesquelles ils arrivent s'en retrouve largement facilitée. En effet, en plus d'être hébergés par leurs proches disposant déjà de logements en Jordanie, ils bénéficient 
également de leurs conseils afin de choisir leurs secteurs de vente et pour s'approvisionner en marchandises dans le royaume.

\section{D'un bout à l'autre d'une chaîne d'approvisionnement mondialisée : la Jordanie...}

Lors des premiers entretiens que j'ai menés auprès des colporteurs de Deir Mqaren récemment installés en Jordanie, j'ai cherché à identifier la provenance exacte des produits qu'ils proposaient à la vente dans les rues de la capitale et les autres villes du nord du royaume. Mis à part un enquêté m'ayant confié continuer à s'approvisionner en Syrie via de coûteuses filières clandestines, tous m'ont affirmé qu'ils en passaient désormais uniquement par Karam, le grossiste jordanien, afin de se procurer leur marchandise. S'agissant précisément des sucreries, elles proviennent de l'usine de confiserie que ce dernier a ouverte en 2000 à Sahab, un quartier industriel du sud-est d'Amman (figure 5).

En effet, cela fait déjà une quinzaine d'années que l'activité de ce grossiste prend en grande partie appui sur celle des colporteurs de Deir Mqaren. Au début des années 1990, lorsque Karam rencontre pour la première fois des vendeurs du village, il est âgé d'une vingtaine d'années et se lance tout juste dans le commerce de petites denrées alimentaires. Un jour qu'il sort de la mosquée du centre-ville, il remarque un stand sur lequel trois hommes proposent des fruits secs, des mokasarat et des malbans ${ }^{13}$. "Comme je me lançais dans la vente de produits similaires, je suis allé voir d'un peu plus près ce qu'ils vendaient. (...) Nous avons échangé sur la provenance et le prix de leurs produits. (...) Je leur ai proposé de leur vendre certains articles à des prix plus intéressants que leurs fournisseurs syriens; et c'est tout simplement ainsi que nous avons commencé à collaborer. Je leur vendais des sucreries et eux me ramenaient en échange des figues séchées, des amandes et des pistaches d'Alep. (...) À la fin des années 1990, j'ai fini par vendre tellement de malbans et de chata matas ${ }^{14}$ aux marchands du village que je me suis décidé à ouvrir ma propre usine de production. (...) Dans les années 2000, environ 500 gars de Deir Mqaren travaillaient dans la vente de sucreries et de mokasarat en Jordanie et au Liban. Avec le roulement qui s'effectuait parmi les vendeurs, il y avait toujours en moyenne une centaine de personnes qui travaillaient dans le pays (...). Avant le début du conflit, les gens du village constituaient près de $80 \%$ de ma clientèle ».

En parallèle de son activité de confection de sucreries, Karam approvisionne également les colporteurs de Deir Mqaren en mokasarat. Afin de connaître le lieu de production de ces fruits à coque, je suis retourné en novembre 2015 dans le magasin des grossistes où s'approvisionne Karam, à Amman, et où j'avais eu l'occasion de me rendre l'année précédente avec lui, alors que nous effectuions ensemble une tournée pour approvisionner quelques marchands de Deir Mqaren résidant dans les environs d'Ajlun, une ville située au nord-ouest de la Jordanie. À l'évocation de son nom, le patron des lieux accepte aussitôt de m'ouvrir les portes de son arrière-boutique, où sept employés - syriens, égyptiens et palestiniens - sont affairés à torréfier des graines de courges et des cacahuètes dans une salle exigüe, dans laquelle règnent la chaleur et le bruit assourdissant des machines en action (figure 7). En interrogeant le personnel de l'établissement, il ne m'a pas fallu longtemps pour retracer l'origine de cette marchandise. Généralement, les noix de cajou proviennent d'Inde, les cacahuètes du 
Soudan, les pistaches d'Iran, les amandes des États-Unis, les noisettes de Turquie, et les graines de courges et de tournesol d'Égypte (figure 8). Il est donc loin le temps où les colporteurs de Deir Mqaren vivaient uniquement de la vente de fruits cultivés dans les limites de leur village. En effet, cette activité de vente ambulante est aujourd'hui en partie insérée dans un marché global, au sein duquel les personnes que j'ai interrogées ne représentent que le dernier maillon d'une longue chaîne d'acteurs et de localités, formant l'ossature d'une des innombrables routes du commerce transnational des fruits à coque.

Figure 7. Atelier de transformation de mokasarat à Amman



Depuis le début de la crise syrienne, c'est d'ici que provient l'essentiel de la marchandise vendue par les colporteurs de Deir Mqaren en Jordanie. 
Figure 8. Origine des fruits à coque vendus par les marchands de Deir Mqaren en Jordanie

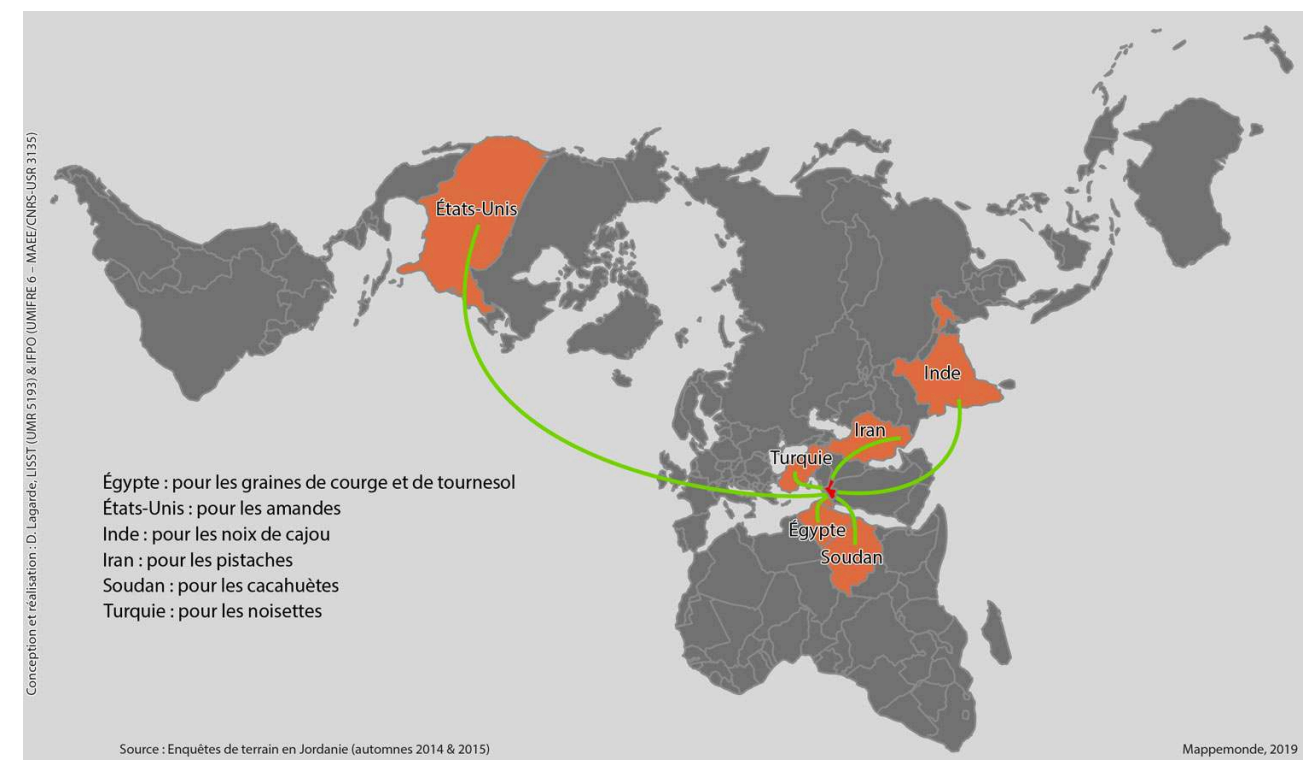

Sources : Entretiens Jordanie ; Réalisation : David Lagarde

\section{... et l'Iran}

Lors d'un séjour en Iran en août 2016, j'ai eu l'occasion de me rendre dans la ville de Kerman, chef-lieu de la province éponyme. Cette localité située sur l'un des segments de l'ancienne route de la soie, au sud-est du pays, est connue depuis le $\mathrm{XV}^{\mathrm{e}}$ siècle pour ses ateliers de confection de tapis persans (Necipoğlu et Leal, 2009, p. 336-337), et plus récemment pour être l'un des principaux centres de production de pistaches sur le marché mondial (figure 9). Avec la levée progressive des sanctions internationales contre le régime iranien, les opportunités commerciales se multiplient ${ }^{15}$ pour les entrepreneurs de la région ayant fait fortune dans l'export de ce fruit à coque particulièrement onéreux. Parallèlement, l'augmentation de la demande chinoise en la matière leur permet de trouver de nouveaux débouchés sur le continent asiatique et d'investir dans d'autres secteurs d'activités, telles que l'import de biens manufacturés produits en Chine, ou bien encore le tourisme haut de gamme en Iran. L'opulence dans laquelle vivent ces chefs d'entreprise de l'industrie agroalimentaire iranienne contraste donc terriblement avec les conditions de vie des "petites mains " qui revendent leur production dans les rues de Jordanie et du Liban (figures 10 et 11). Ironie de ce négoce international, il est aussi surprenant de constater que ce commerce amène des réfugiés syriens majoritairement opposés au régime de Bachar al-Assad à acheter, sans même le savoir $^{16}$, des denrées produites en Iran, un pays constituant l'un des principaux soutiens économique et militaire de l'actuel gouvernement syrien. 
Figure 9. Usine de conditionnement de pistaches dans la ville de Kerman (Iran)

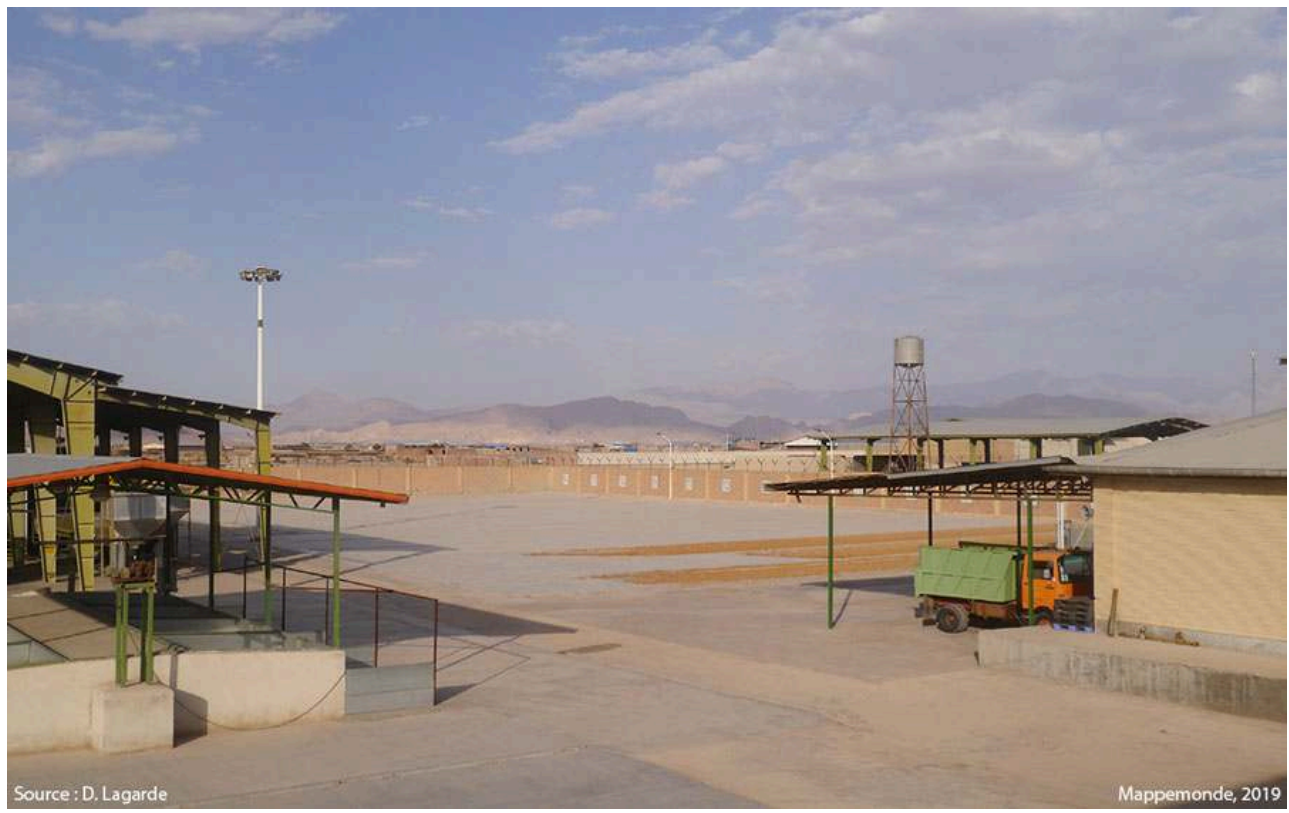

D. Lagarde

Figure 10. Vendeurs ambulants proposant leurs marchandises sur le parvis de la mosquée du centre-ville d'Amman

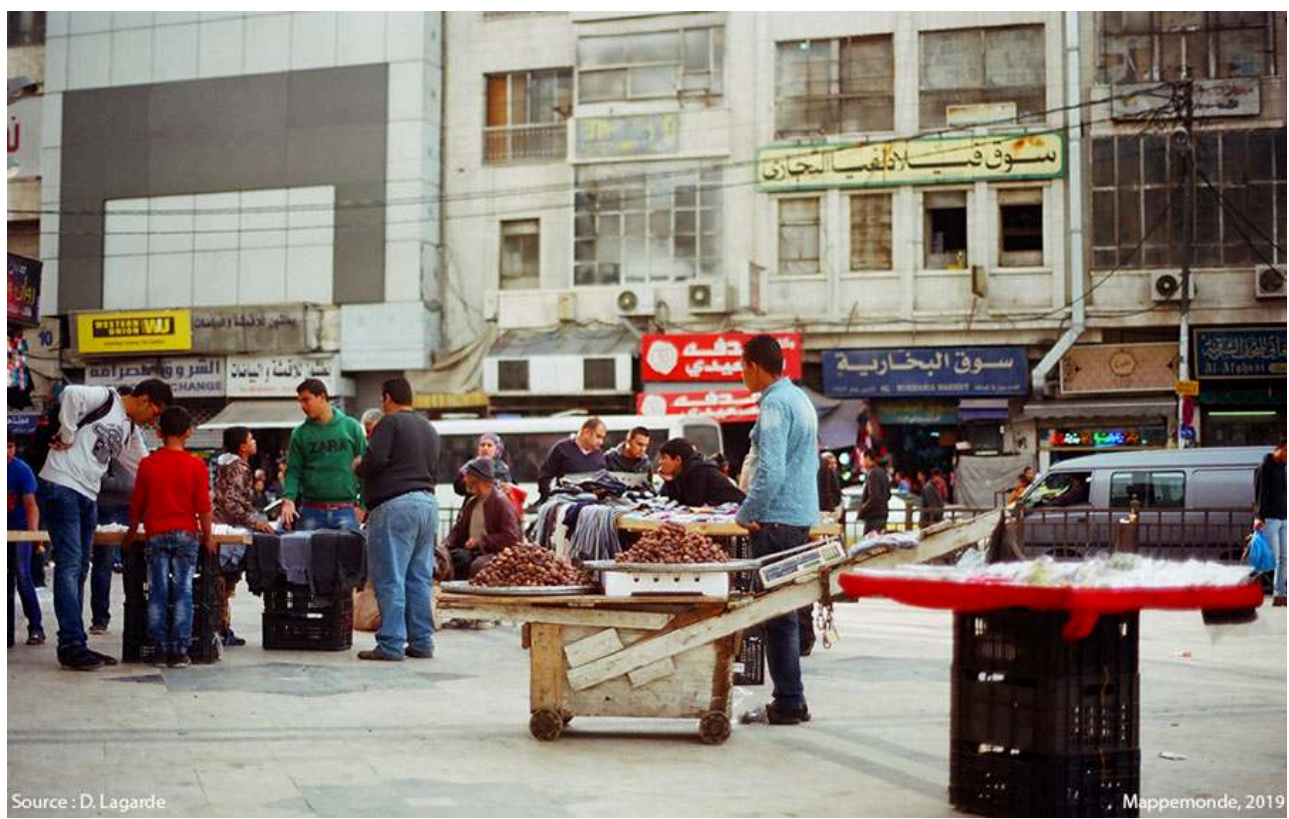

D. Lagarde 
Figure 11. Stand d'un marchand de Deir Mqaren dans la gare routière de Raghdan à Amman

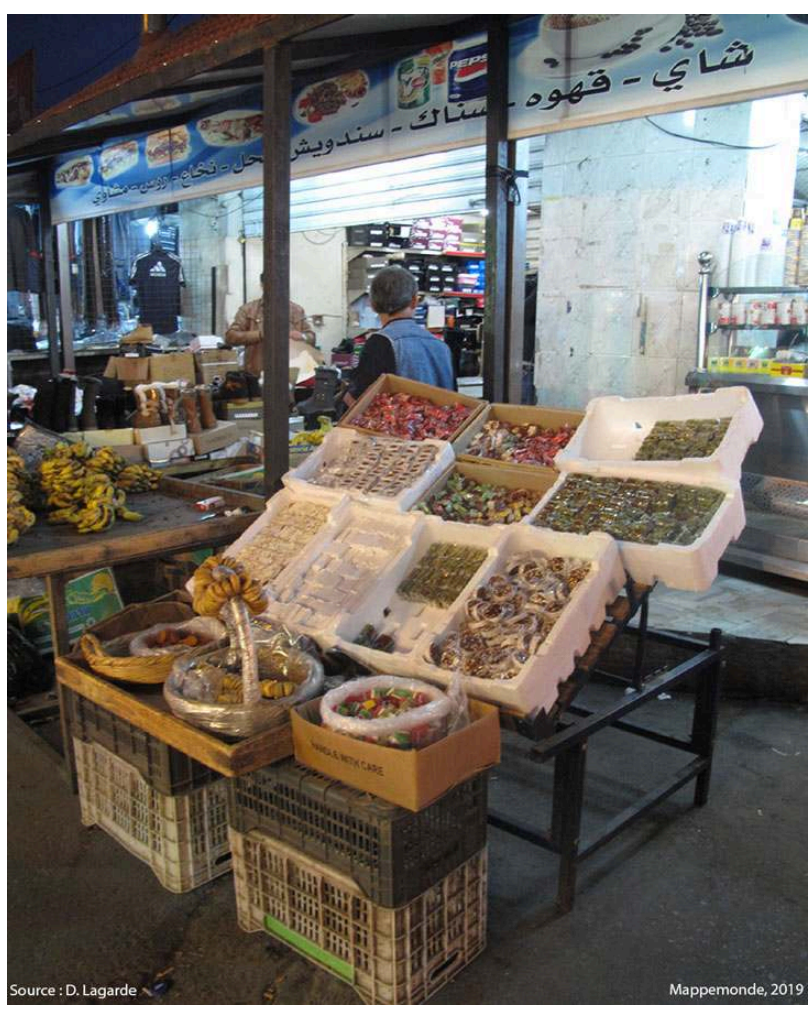

D. Lagarde

\section{Conclusion}

Comme cette contribution permet de l'entrevoir, l'activité de colportage fait partie intégrante de l'identité collective des villageois de Deir Mqaren. À ce titre, les enquêtés ont régulièrement insisté sur la force physique et les talents commerciaux hérités de leurs ancêtres qui exerçaient un travail similaire par le passé, au point que ces caractéristiques seraient, pour reprendre leurs propres mots, « inscrites dans l'ADN des enfants du village ». Si nous laissons de côté l'hypothèse de l'évolution génétique, il ne fait en revanche aucun doute que la transmission d'une culture de la mobilité, acquise grâce à la perpétuation de ces pratiques marchandes depuis l'époque ottomane, s'est révélée être une ressource considérable pour plusieurs générations d'habitants de Deir Mqaren. En faisant évoluer le tracé de leurs itinéraires ainsi que leurs sources d'approvisionnement afin de s'adapter aux contraintes structurelles auxquelles ils se sont retrouvés confrontés à plusieurs reprises au cours du $\mathrm{XX}^{\mathrm{e}}$ siècle (figure 12), ces paysans colporteurs de la vallée du Barada ont toujours réussi à pallier la pénurie d'emplois à l'échelle locale, en allant se procurer ailleurs des revenus inaccessibles localement, tout en venant se greffer sur des filières d'approvisionnement mondialisées, ceci après avoir produit durant plusieurs décennies leur propre marchandise. 
Figure 12. Temporalités de l'activité de vente ambulante à Deir Mqaren



Conception et réalisation : David Lagarde. Sources : enquêtes de terrain en Jordanie (automnes 2014 et 2015)

Depuis 2011, la guerre en Syrie a profondément affecté le réseau marchand et migratoire de ces colporteurs, ainsi que leurs conditions de travail (Lagarde et Doraï, 2017). Les colporteurs n'étant plus en mesure de générer des bénéfices sur les différences de prix transfrontalières comme ils le faisaient avant le début de la crise, tout en se voyant désormais contraints de s'approvisionner uniquement en Jordanie, leurs revenus ont connu une baisse drastique. Dans ce contexte, plusieurs marchands ont préféré abandonner leur activité pour tenter de reconstruire leur vie loin du Proche-Orient. Bien qu'étant extrêmement minoritaires dans la population totale des exilés du village, majoritairement réfugiée au Liban et en Jordanie, certains (anciens) colporteurs résident donc désormais au Canada, aux États-Unis, en Suède et surtout en Allemagne. Ainsi, on peut s'interroger sur l'avenir de ce réseau marchand, et plus généralement sur la relation qui lie les villageois de Deir Mqaren au commerce de menues denrées alimentaires. Les évènements géopolitiques récents observés au Proche-Orient vont-ils se traduire par une disparition pure et simple de cette activité, ou bien au contraire donner lieu à une nouvelle reconfiguration de ce dispositif réticulaire? Si ce commerce perdure, quels changements sociaux, économiques et spatiaux est-il susceptible de connaitre à l'avenir ? Cela en passera-t-il une nouvelle fois par la connexion à de nouvelles filières d'approvisionnement? Au regard du caractère particulièrement mobile et du grand esprit d'initiative dont ont toujours fait preuve les habitants de cette bourgade de Syrie, il ne serait guère surprenant que leur dispersion spatiale engendre, à moyen terme, de nouvelles pratiques marchandes entre les différents pôles d'installation des exilés de Deir Mqaren, donnant ainsi lieu à un élargissement géographique du cadre de leurs échanges. Toujours est-il que la résilience et la capacité d'adaptation dont continuent de faire preuve les exilés de Deir Mqaren dans un contexte de crise méritent largement d'être soulignées, ne serait-ce que pour relativiser les visions institutionnelles et globalisantes ayant trop souvent tendance à dépeindre les réfugiés comme les «victimes passives d'un sort qui les dépasse » (Monsutti, 2004). 


\section{BIBLIOGRAPHIE}

BALANCHE F. (2006). « Damas : chronique d'une pénurie annoncée ». Confluences Méditerranée, vol. 58, n 3, p. 91-101.

BALANCHE F. (2009). «L'habitat illégal dans l'agglomération de Damas et les carences de l'État ». Revue géographique de l'Est, vol. 49, nº 4. En ligne : https://journals.openedition.org/rge/1980 BIANQUIS A.-M. (1977). « Le problème de l'eau à Damas et dans sa Ghouta ». Géocarrefour, vol. 52, n ${ }^{\circ}$ 1, p. 35-53.

BouHALi A. (2015). « Médina J'dida (Oran), un quartier-marché sur les routes algériennes du commerce transnational ». Les Cahiers d'EMAM. Études sur le Monde arabe et la Méditerranée, $\mathrm{n}^{\circ} 26$. En ligne : https://journals.openedition.org/emam/889

ChATEL F. DE (2014). “The Death of the Garden of Eden”. In TVEDT T. et OESTIGAARD T.(dir.), History of Water, Londres : I. B. Tauris.

CHÂTEL F., RABA'A M. (2014). “Waterless Wadi Barada: Manufacturing scarcity in a Syrian River Valley”. Middle East Report, vol. 44, p. 10-17.

CHOPLIN A., PLIEZ O. (2015). “The Inconspicuous Spaces of Globalization”. Articulo. Journal of Urban Research, $\mathrm{n}^{\circ} 12$. En ligne : https://journals.openedition.org/articulo/2905

CHOPLIN A., PLIEZ O. (2016). « Des mondialisations plus discrètes ». La Vie des idées. En ligne : https:// laviedesidees.fr/Des-mondialisations-plus-discretes.html

CHOPLIN A., PLIEZ O. (2018). La mondialisation des pauvres. Loin de Wall St. et de Davos. Paris : Seuil.

CORTES G. (1998). « Migrations, systèmes de mobilité, espaces de vie : à la recherche de modèles ». L'Espace géographique, vol. 27, n 3, p. 265-275.

DERRIENNIC J.-P. (1974). Israël en guerre. Paris : Presses de Sciences Po.

DESCAMPS F. (2006). Les sources orales et l'histoire : récits de vie, entretiens, témoignages oraux. Paris : Éditions Bréal.

DORON A. (2015). « De la marge au monde : la structuration mouvementée d'une place marchande transnationale à Ben Gardane (Tunisie) ». Les Cahiers d'EMAM. Études sur le Monde arabe et la Méditerranée, $\mathrm{n}^{\circ}$ 26. En ligne : https://journals.openedition.org/emam/1065

GEHRIG T., MONSUTTI A. (2003). « Territoires, flux et représentations de l'exil afghan : le cas des Hazaras et des Kaboulis ». A contrario, vol. 1, n 1, p. 61-78.

LAGARDE D. (2019, à paraître). « Du refuge moyen-oriental à la dispersion mondiale. Structuration de l'exode syrien entre 2011 et 2016 ». Revue européenne des migrations internationales, vol. 35, $\mathrm{n}^{\circ} 3$ $\& 4$.

LAGARDE D. (2018). Sur les routes de l'exil syrien. Récits de vie et parcours migratoires des réfugiés de Deir Mqaren. Thèse de doctorat en Géographie. Université de Toulouse Jean Jaurès.

LAGARDE D., DORAÏ K. (2017). « De la campagne syrienne aux villes jordaniennes. Un réseau marchand transfrontalier à l'épreuve du conflit syrien ». Espace, Populations, Sociétés, $\mathrm{n}^{\circ} 2$. En ligne : https://journals.openedition.org/eps/7212

LONGUENESSE É. (1979). « Monde paysan et industrialisation en Syrie ». Géocarrefour, vol. 54, n 3, p. 249-255. 
MA MUNG E. (2009). « Le point de vue de l'autonomie dans l'étude des migrations internationales : “penser de l'intérieur" les phénomènes de mobilité ». In DUREAU F. et HILY M.-A. (dir.), Les mondes de la mobilité, Presses de l'Université de Rennes, p. 25-38.

MISSAOUI L., TARRIUS A. (2006) «Villes et migrants, du lieu-monde au lieu-passage ». Revue européenne des migrations internationales, vol. 22, $\mathrm{n}^{\circ} 2$, p. 43-65. En ligne : https:// journals.openedition.org/remi/2818

MONSUTTI A. (2004). Guerres et migrations : réseaux sociaux et stratégies économiques des Hazaras d'Afghanistan. Neuchâtel/Paris : Institut d'ethnologie/Maison des sciences de l'Homme. NECIPOĞLU G., LEAL K. A. (2009). Muqarnas. BRILL.

PICARD É. (2013). « La Syrie de 1946 à 1979 ». In RAYMOND A. (dir.), La Syrie d'aujourd'hui, Aix-enProvence : Institut de recherches et d'études sur les mondes arabes et musulmans, p. 143-184.

PLIEZ O. (2007). « Des jeans chinois dans les rues du Caire, ou les espaces discrets de la mondialisation ». M@ppemonde, nº 88-4. En ligne : https://mappemonde-archive.mgm.fr/num16/ articles/art07404.html

PLIEZ O. BELGUidoum S. (2012). «Construire une route de la soie entre l'Algérie et la Chine ». Diaspora. Histoire et sociétés, $\mathrm{n}^{\circ}$ 20, p. 115-130.

PUIG C. (2005). « La place des fruits en Méditerranée nord occidental à partir des actes de la pratique et des tarifs marchands (XII ${ }^{e}$-Première moitié XIV ${ }^{\mathrm{e}}$ siècles) ». Archéologie du Midi médiéval, t. 23-24, p. 119-128.

REIFFERS J.-L., éd. (1997). La Méditerranée aux portes de l'an 2000. Paris : Economica. RICHMOND A. H. (1993). "Reactive Migration: Sociological Perspectives On Refugee Movements". Journal of Refugee Studies, vol. 6, n 1, p. 7-24.

SEURAT M. (1979). « État et paysans en Syrie ». Géocarrefour, vol. 54, n 3, p. 257-270.

TARRIUS A. (1996). « Territoires circulatoires des migrants et espaces européens ». In HIRSCHORN M. et BERTHELOT J.-M. (dir.), Mobilités et ancrages. Vers un nouveau mode de spatialisation? Paris :

L'Harmattan, p. 93-100.

THOUMIN R. (1936). Géographie humaine de la Syrie centrale. Librairie Ernest Leroux.

\section{NOTES}

1. Les mokasarat sont un mélange de fruits à coque et de graines torréfiés et salés.

2. Emmanuel Ma-Mung définit l'autonomie de la manière suivante : «L'autonomie comme savoirfaire pourrait être alors définie comme la capacité d'un sujet, individuel ou collectif, à constituer et maintenir un monde, et l'autonomie comme pouvoir-faire comme la capacité de ce même sujet à agir sur et à transformer ce monde » (Ma-Mung, 2009, p. 29).

3. Notons ici que la vente ambulante en Jordanie, au Liban ou bien même en Syrie constitue une activité exclusivement masculine. En effet, aucune femme de Deir Mqaren n'a un jour exercé le métier de colporteuse.

4. Parmi les 24 enquêtés, 8 ont été interrogés au moins à deux reprises en Jordanie (et pour 3 d'entre eux également en Allemagne), entre octobre 2014 et juin 2016.

5. Selon l'Organisation des Nations unies pour l'alimentation et l'agriculture (FAO), la Turquie a produit 675000 tonnes de noisettes en 2017, contre 131281 pour l'Italie, deuxième producteur mondial. 
6. Le Bilad esh-Sham est le nom donné à la région contenant les États actuels de Syrie, de Jordanie, d'Israël, de Palestine et du Liban, entité autrefois économiquement et culturellement homogène, dominée par la ville de Damas.

7. Tous les prénoms mentionnés dans cet article ont été anonymisés.

8. Sorte de grande besace, constituée de deux poches profondes, portée sur l'épaule en bandoulière.

9. Arrivé à la tête de la Syrie en novembre 1970 à la suite d'un Coup d'État, Hafez al-Assad se maintient au pouvoir jusqu'à sa mort en juin 2000. Depuis, c'est son fils Bachar qui règne en maître sur ce pays.

10. Dans le cas damascène, le terme de Ghouta fait référence à la zone où étaient situées des terres agricoles à l'est de la capitale et qui, pendant des siècles, ont constitué une oasis luxuriante au milieu du désert.

11. Depuis la fin de l'époque mandataire, où Deir Mqaren comptait 599 âmes selon un décompte effectué par le Service Géographique des Forces Françaises du Levant, cette bourgade a connu une très forte croissance démographique, puisque selon les enquêtés, environ 5000 personnes y résidaient en 2011. Cette augmentation est essentiellement le fait d'une forte baisse du taux de mortalité observée dans l'ensemble de la Syrie depuis le tournant du XXe siècle (Reiffers, 1997). En effet, même si quelques familles extérieures au village se sont installées à Deir Mqaren au cours de cette période, ce phénomène reste marginal et ne permet pas à lui seul d'expliquer une telle augmentation de population.

12. Lors de notre entretien, Karam n'a pas été en mesure de spécifier quels étaient les autres pays de provenance de ces marchandises.

13. Confiserie ressemblant peu ou prou à une sorte de nougat mou et de pâte de fruit, fabriquée à partir de jus de raisin et généralement fourrée de fruits à coque, le plus souvent des noix ou des pistaches. Afin d'offrir un produit meilleur marché aux consommateurs les plus pauvres, les cacahuètes sont de plus en plus souvent utilisées dans sa confection. C'est notamment le cas des malbans qui sont produits dans l'usine de Karam.

14. Les chata matas sont de gros bonbons acidulés (bourrés de colorants) produits sous forme de grandes plaques qui sont ensuite découpées en cubes d'environ deux centimètres d'épaisseur.

15. Il est possible que les débouchés commerciaux de ces exportateurs iraniens aient été sensiblement affectés par le retour des sanctions américaines contre l'Iran en 2018, et par là même le relatif dynamisme économique qu'ont connu leurs entreprises ces dernières années.

16. Notons que lorsque j'ai tenté de questionner les vendeurs de Deir Mqaren sur l'origine des mokasarat qu'ils revendent en Jordanie, aucun d'entre eux n'a été en mesure de répondre à mes interrogations. Tous m'ont renvoyé vers Karam, affirmant que celui-ci serait mieux placé pour m'éclairer sur le sujet.

\section{RÉSUMÉS}

En étudiant les mobilités de colporteurs syriens, cet article entend souligner la capacité d'adaptation d'une communauté rurale soumise aux vicissitudes des crises géopolitiques procheorientales. Leur histoire, les reconfigurations régulières de leur réseau marchand, ainsi que l'origine des produits alimentaires qu'ils vendent dans les rues de Jordanie, révèlent en filigrane une facette peu visible de la mondialisation, qui contribue à relier entre elles des villes et des 
bourgades des Suds, situées à l'écart des grands centres décisionnels et économiques les plus médiatisés de la planète.

By studying the mobility of Syrian peddlers, this article aims to highlight the adaptability of a rural community subject to the vicissitudes of geopolitical crises in the Middle East. Their history, the regular reconfigurations of their trade network, as well as the origin of the food products they sell in the streets of Jordan, reveal a hidden facet of globalization. This aspect helps connect the cities and towns of the Global South that are far removed from the most publicised political and financial centers in the world.

Estudiando la movilidad de los vendedores ambulantes sirios se comprende la adaptación de las comunidades rurales ante las vicisitudes derivadas de las crisis geopolíticas en Oriente Medio. La cara oculta de la globalización se visibiliza a través de sus historias, de las reconfiguraciones periódicas de sus redes comerciales, o del origen de los alimentos que venden en las calles de Jordania. Al mismo tiempo conectan las ciudades con los núcleos rurales y diseminados del Sur que se encuentran alejado de los grandes centros económicos y decisorios del planeta.

\section{INDEX}

Thèmes : Les routes de la soie existent déjà. Routes transnationales et places marchandes du made in China entre Asie Afrique et Europe

Mots-clés : colportage, commerce transfrontalier, migrations, mondialisation

Keywords : cross-border trade, globalization, migration, street-peddling

Palabras claves : venta ambulante, comercio transfronterizo, migraciones, globalización

\section{AUTEUR}

\section{DAVID LAGARDE}

Post-doctorant CNRS, équipe LISST (UMR 5193). Membre associé à l'Institut français du ProcheOrient (UMIFRE 6 - MAEE/CNRS-USR 3135) 